\title{
MALDI-TOF: Introduction in routine of a system for the rapid identification of microorganisms
}

\author{
Chiara Vismara, Maria Chiara Sironi, Monica Drago, Giuseppina Cafiero, Liana Asti, \\ Chiara Grimaldi, Gianluigi Lombardi \\ S.C Microbiologia e Virologia, Dipartimento di Medicina di Laboratorio, A.O. Ospedale Niguarda Ca' Granda Milano
}

Key words: MALDI-TOF, identification, bacteria

MALDI-TOF: Introduzione in routine di un sistema di identificazione rapida

\section{SUMMARY}

Introduction. MALDI-TOF (Matrix Assisted Laser Desorption/lonization-Time of Flight) is an recently introduced technique for the rapid identification of microorganisms by mass spectrometry $(I, 3)$ (Fig.I). The aim of this work was the evaluation and validation of the MALDI-TOF method (Bruker Daltonics) for inclusion in the routine workflow of a clinical microbiology laboratory.

Methods. We analyzed strains isolated from different clinical specimens. For each species of microorganism we calculated the percentage of agreement by comparing the identification of MALDI-TOF with that obtained using the methods currently in use in the laboratory. In case of discordant results the conclusive identification was carried out by sequencing of $16 \mathrm{~S}$ ribosomal DNA (Tab.3) using the MicroSeq identification system 500 (Applied Biosystems) (2). Alpha-hemolytic streptococci were instead identified by the ATB galleries (BioMerieux). ATCC strains were used as Quality Control.

Results. I 340 strains were analyzed, including 479 enterobacteria, 188 gram-negative bacilli, 189 staphylococci, I 40 enterococci, 84 Haemophilus spp, I I 4 streptococci, 40 yeasts and 106 less common microorganisms. Gramnegative bacteria, enterococci, Staphylococcus aureus, beta-hemolytic streptococci and yeasts showed full agreement (Tab.I e Tab.2). In addition, MALDI-TOF has proved a reliable method for identification of fastidious germs such as Legionella, Branhamella, Neisseriae, Listeria monocytogenes, Corynebacteria, and anaerobes. Alpha-hemolytic streptococci were but in most cases identified as S. pneumoniae.

Conclusions. The identification by mass spectrometry allows to obtain reliable results in minutes for most of the organisms isolated from the routine. Considerable importance for the performance of the system plays the quality of the database in the instrument. The high percentage of concordance between identification with the standard methods and MALDI-TOF allowed the introduction of this method in routine workflow with the exception of alpha hemolytic streptococci for which the current system of identification is still in use.

\section{INTRODUZIONE}

MALDI-TOF (Matrix Assisted Laser Desorption/Ionization-Time of Flight) è una tecnica di analisi in spettrometria di massa recentemente introdotta per l'identificazione rapida dei microrganismi (1,3) (Fig.1). Obiettivo di questo lavoro è stato la valutazione e validazione dello strumento MALDI-TOF (Bruker Daltonics) per l'inserimento in routine in un laboratorio di microbiologia clinica.

\section{METODI}

Sono stati analizzati ceppi isolati da materiali biologici diversi. Per ogni specie di microrganismo si è calcolata la percentuale di concordanza, confrontando l'identificazione di MALDI-TOF con quella ottenuta mediante le metodiche attualmente in uso presso il laboratorio. In caso di risultati discordanti si è proceduto ad identificazione con- clusiva mediante sequenziamento del 16S DNA ribosomale (Tab.3) utilizzando il sistema di identificazione MicroSeq 500 (Applied Biosystems) (2). Gli streptococchi alfa emolitici sono stati invece identificati mediante le gallerie ATB (bioMérieux). Come Controllo di Qualità sono stati utilizzati ceppi ATCC.

\section{RISULTATI}

Sono stati analizzati 1340 ceppi, 479 enterobatteri, 188 Gram negativi non fermentanti, 189 stafilococchi, 140 enterococchi, 84 emofili, 114 streptococchi, 40 lieviti, 106 microrganismi a minore frequenza di isolamento. Batteri Gram-negativi, enterococchi, S. aureus, streptococchi beta emolitici e lieviti hanno evidenziato piena concordanza (Tab.1 e Tab.2). MALDI-TOF si è dimostrato una metodica affidabile anche per l'identificazione di germi difficili quali Legionella, Branhamella,

\section{Corresponding author: Vismara Chiara}

S.C Microbiologia e Virologia, Dipartimento di Medicina di Laboratorio, A.O. Ospedale Niguarda Ca' Granda

Piazza Ospedale Maggiore, 3 - 20162 Milano - Tel.: 0264443888 - Fax.: 0264442916

E-mail: chiara.vismara@ospedaleniguarda.it 
Tabella I. Percentuali di concordanza tra sistema MALDI_TOF e metodica classica nei patogeni Gram-positivi.

\begin{tabular}{|c|c|c|c|c|c|c|}
\hline & & \multicolumn{2}{|c|}{$\begin{array}{c}\text { ID } \\
\text { NON CORRETTA }\end{array}$} & \multicolumn{2}{|c|}{$\begin{array}{c}\text { ID } \\
\text { CORRETTA }\end{array}$} & \multirow{2}{*}{$\begin{array}{c}\text { TOTALE } \\
\text { ID } \\
\mathbf{N}\end{array}$} \\
\hline & & $\mathbf{N}$ & $\%$ & $\mathbf{N}$ & $\%$ & \\
\hline Staphylococcus aureus & Staphylococcus aureus & & $0 \%$ & 100 & $100 \%$ & 100 \\
\hline \multirow[t]{10}{*}{ STCM } & Staphylococcus epidermidis & 1 & $2 \%$ & 60 & $98 \%$ & 61 \\
\hline & Staphylococcus haemolyticus & & $0 \%$ & 13 & $100 \%$ & 13 \\
\hline & Staphylococcus warneri & 2 & $67 \%$ & 1 & $33 \%$ & 3 \\
\hline & Staphylococcus simulans & & $0 \%$ & 3 & $100 \%$ & 3 \\
\hline & Staphylococcus lugdunensis & & $0 \%$ & 3 & $100 \%$ & 3 \\
\hline & Staphylococcus hominis & & $0 \%$ & 2 & $100 \%$ & 2 \\
\hline & Staphylococcus auricolaris & 1 & $100 \%$ & & $0 \%$ & 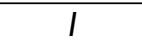 \\
\hline & Staphylococcus saprophyticus & & $0 \%$ & I & $100 \%$ & I \\
\hline & Staphylococcus schleiferi & & $0 \%$ & 1 & $100 \%$ & 1 \\
\hline & Staphylococcus capitis & & $0 \%$ & 1 & $100 \%$ & $I$ \\
\hline STCM Totale & & 4 & $4 \%$ & 85 & $96 \%$ & 89 \\
\hline \multirow[t]{4}{*}{ Enterococchi } & Enterococcus faecalis & & $0 \%$ & 103 & $100 \%$ & 103 \\
\hline & Enterococcus faecium & & $0 \%$ & 30 & $100 \%$ & 30 \\
\hline & Enterococcus avium & & $0 \%$ & 6 & $100 \%$ & 6 \\
\hline & Enterococcus gallinarum & & $0 \%$ & 1 & $100 \%$ & 1 \\
\hline Enterococchi Totale & & & $0 \%$ & 140 & $100 \%$ & 140 \\
\hline \multirow[t]{11}{*}{ Streptococchi } & Streptococcus agalactiae & & $0 \%$ & 44 & $100 \%$ & 44 \\
\hline & Streptococcus pyogenes & & $0 \%$ & 30 & $100 \%$ & 30 \\
\hline & Streptococcus constellatus & & $0 \%$ & 11 & $91 \%$ & 11 \\
\hline & Streptococcus dysgalactiae & & $0 \%$ & 8 & $100 \%$ & 8 \\
\hline & Streptococcus anginosus & & $0 \%$ & 5 & $100 \%$ & 5 \\
\hline & Streptococcus mitis & 4 & $100 \%$ & & $0 \%$ & 4 \\
\hline & Streptococcus gallolyticus & & $0 \%$ & 4 & $100 \%$ & 4 \\
\hline & Streptococcus oralis & 3 & $100 \%$ & & $0 \%$ & 3 \\
\hline & Streptoccus sanguinis & 2 & $100 \%$ & & $0 \%$ & 2 \\
\hline & Streptococcus salivarius & & $0 \%$ & 2 & $100 \%$ & 2 \\
\hline & Streptococcus pneumoniae & & $0 \%$ & 1 & $100 \%$ & 1 \\
\hline Streptococchi Totale & & 9 & $8 \%$ & 105 & $92 \%$ & I/4 \\
\hline
\end{tabular}

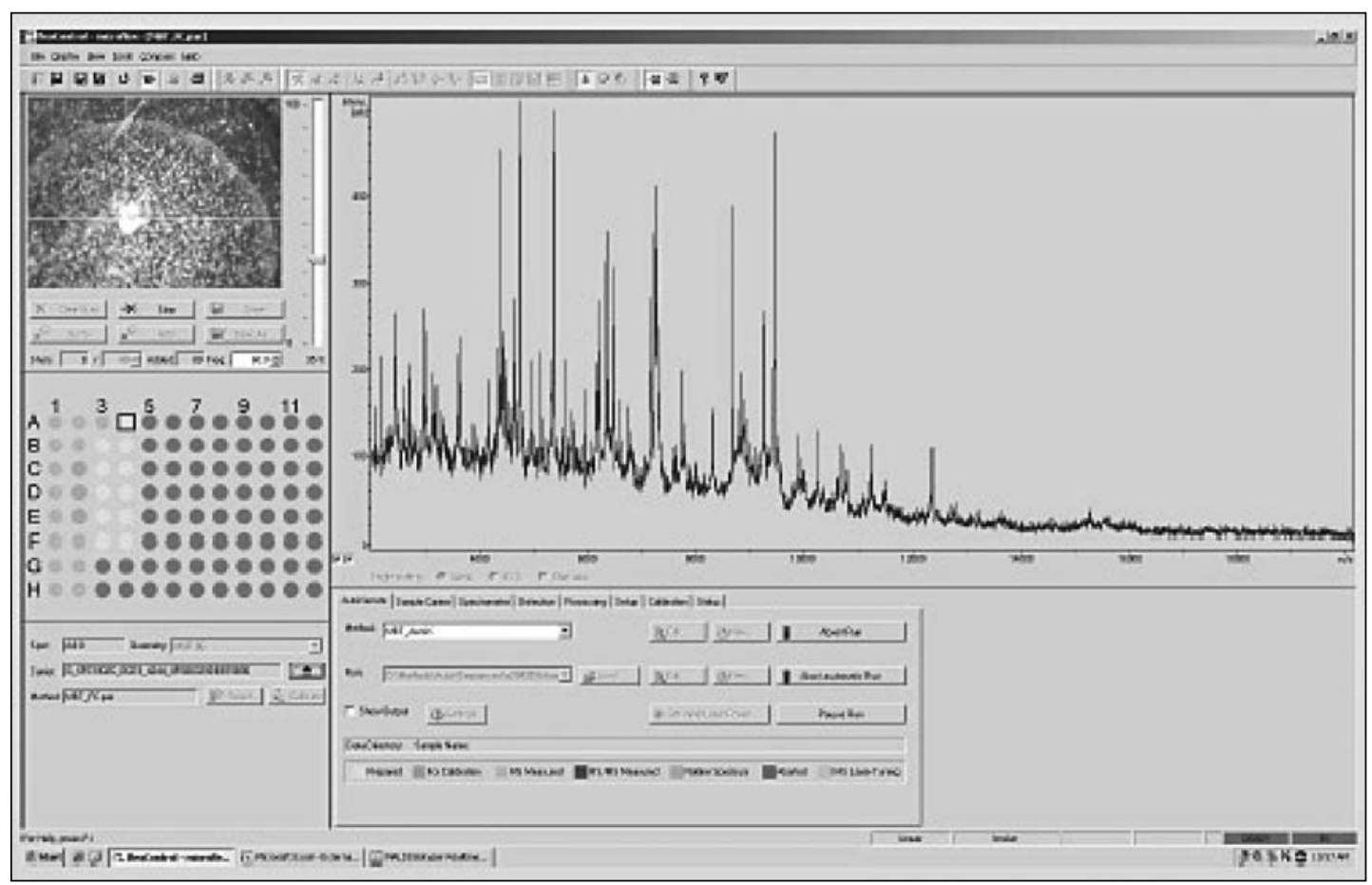

Figura I. Esempi di letture eseguite da MALDI-TOF) relativamente a un ceppo di Staphylococcus aureus. 
Tabella 2. Percentuali di concordanza tra sistema MALDI-TOF e metodica classica nei patogeni Gram-negativi.

\begin{tabular}{|c|c|c|c|c|c|c|}
\hline & & \multicolumn{2}{|c|}{$\begin{array}{c}\text { ID } \\
\text { NON CORRETTA }\end{array}$} & \multicolumn{2}{|c|}{$\begin{array}{c}\text { ID } \\
\text { CORRETTA }\end{array}$} & \multirow{2}{*}{$\begin{array}{c}\text { TOTALE } \\
\text { ID } \\
\mathbf{N}\end{array}$} \\
\hline & & $\mathbf{N}$ & $\%$ & $\mathbf{N}$ & $\%$ & \\
\hline \multirow[t]{16}{*}{ Enterobatteri } & Escherichia coli & & $0 \%$ & 173 & $100 \%$ & 173 \\
\hline & Klebsiella pneumoniae & & $0 \%$ & 126 & $100 \%$ & 126 \\
\hline & Proteus mirabilis & & $0 \%$ & 54 & $100 \%$ & 54 \\
\hline & Enterobacter cloacae & & $0 \%$ & 48 & $100 \%$ & 48 \\
\hline & Serratia marcescens & & $0 \%$ & 19 & $100 \%$ & 19 \\
\hline & Morganella morganii & & $0 \%$ & 14 & $100 \%$ & 14 \\
\hline & Citrobacter freundii & & $0 \%$ & 10 & $100 \%$ & 10 \\
\hline & Klebsiella oxytoca & & $0 \%$ & 10 & $100 \%$ & 10 \\
\hline & Enterobacter aerogenes & & $0 \%$ & 8 & $100 \%$ & 8 \\
\hline & Citrobacter koseri & & $0 \%$ & 6 & $100 \%$ & 6 \\
\hline & Salmonella sp & & $0 \%$ & 3 & $100 \%$ & 3 \\
\hline & Hafnia alvei & & $0 \%$ & 2 & $100 \%$ & 2 \\
\hline & Providencia rettgeri & & $0 \%$ & 2 & $100 \%$ & 2 \\
\hline & Proteus vulgaris & & $0 \%$ & 2 & $100 \%$ & 2 \\
\hline & Providencia stuartii & & $0 \%$ & $I$ & $100 \%$ & 1 \\
\hline & Serratia liquefaciens & & $0 \%$ & l & $100 \%$ & l \\
\hline Enterobatteri Totale & & & $0 \%$ & 479 & $100 \%$ & 479 \\
\hline \multirow[t]{2}{*}{ GN Non fermentanti } & Pseudomonas aeruginosa & & $0 \%$ & 85 & $100 \%$ & 85 \\
\hline & Stenotrophomonas maltophilia & 7 & $32 \%$ & 15 & $68 \%$ & 22 \\
\hline GN Non ferm. Totale & & 7 & $7 \%$ & 100 & $93 \%$ & 107 \\
\hline \multirow[t]{8}{*}{ Altri GN } & Acinetobacter baumannii & 3 & $5 \%$ & 60 & $95 \%$ & 63 \\
\hline & Moraxella catarrhalis & & $0 \%$ & 9 & $100 \%$ & 9 \\
\hline & Pasteurella multocida & & $0 \%$ & 2 & $100 \%$ & 2 \\
\hline & Acinetobacter johnsonii & & $0 \%$ & 2 & $100 \%$ & 2 \\
\hline & Aeromonas hydrophila & & $0 \%$ & 2 & $100 \%$ & 2 \\
\hline & Achromobacter denitrificans & $I$ & $100 \%$ & & $0 \%$ & $I$ \\
\hline & Neisseria gonorrhoeae & & $0 \%$ & I & $100 \%$ & l \\
\hline & Achromobacter xylosoxidans & & $0 \%$ & 1 & $100 \%$ & I \\
\hline Altri GN Totale & & 4 & $5 \%$ & 77 & $95 \%$ & 81 \\
\hline
\end{tabular}

Tabella 3. Identificazione di alcune specie mediante 3 metodi

\begin{tabular}{lll}
\hline Id Convenzionale & MALDI-TOF & I 6S RNA sequencing \\
\hline Acinetobacter baumannii complex & Acinetobacter genomospecies & Acinetobacter genomospecies \\
\hline S. chromogenes & S. lugdunensis & S. lugdunensis \\
\hline C. jeikeium & C. afermentans & C. afermentans \\
\hline Finegoldia magna & Peptostreptococcus micros & Finegoldia magna \\
\hline
\end{tabular}

Neisseriae, Lysteria monocytogenes, anaerobi e corinebatteri. Gli streptococchi alfa emolitici sono stati invece identificati nella maggior parte dei casi come $S$. pneumoniae.

\section{CONCLUSIONI}

L'identificazione in spettrometria di massa permette di ottenere in pochi minuti risultati affidabili per la maggior parte dei microrganismi isolati dalla routine. Notevole importanza per le performance del sistema riveste la qualità del database presente nello strumento. L'elevata percentuale di concordanza tra identificazione con i metodi standard e MALDI-TOF ha permesso l'introduzione in routine della metodica ad eccezione degli Streptococchi alfa emolitici per i quali si è quindi mantenuto l'attuale sistema di identificazione.

\section{BIBLIOGRAFIA}

1. Bizzini A., Durussel C., Bille J., Greub G., and Prod'hom G. Performance of Matrix-Assisted Laser Desorption Ionization-Time of Flight Mass Spectrometry for Identification of Bacterial Strains Routinely Isolated in a Clinical Microbiology Laboratory. $J$ of Clin Microbiol May 2010; 48 (5): 1549-1554

2. Bizzini A., Jaton K., Romo D., Bille J., Prod'hom G., and Greub G. Matrix-Assisted Laser Desorption Ionization - Time of Flight Mass Spectrometry as an Alternative to $16 \mathrm{~S}$ rRNA Gene Sequencing for Identification of Difficult-To-Identify Bacterial Strains. J of Clin Microbiol Feb. 2011; 49 (2): 693696.

3. Risch M., Radjenovic D., Han J.N., Wydler M., Nydegger U., Risch L. Comparison of MALDI TOF with conventional identification of clinically relevant bacteria. Swiss Med Wkly 2010; 140: w13095. 